\title{
On the least quadratic non-residue
}

\author{
Y.-K. LAU \& J. WU
}

\begin{abstract}
We prove that for almost all real primitive characters $\chi_{d}$ of modulus $|d|$, the least positive integer $n_{\chi_{d}}$ at which $\chi_{d}$ takes a value not equal to 0 and 1 satisfies $n_{\chi_{d}} \ll \log |d|$, and give a quite precise estimate on the size of the exceptional set. Also, we generalize Burgess' bound for $n_{\chi_{p^{\prime}}}$ (with $p^{\prime}$ being a prime up to \pm sign) to composite modulus $|d|$ and improve Garaev's upper bound for the least quadratic non-residue in Pajtechiı-S̆apiro's sequence.
\end{abstract}

\section{$\S$ 1. Introduction}

Let $q \geq 2$ be an integer and $\chi$ a non principal Dirichlet character modulo $q$. Here the evaluation of the least integer $n_{\chi}$ among all positive integers $n$ for which $\chi(n) \neq 0,1$ is referred as Linnik's problem. In case $\chi$ coincides with the Legendre symbol, $n_{\chi}$ is a least quadratic non-residue. Concerning the size of $n_{\chi}$, Pólya-Vinogradov's inequality

$$
\max _{x \geq 1}\left|\sum_{n \leq x} \chi(n)\right| \ll q^{1 / 2} \log q
$$

implies trivially $n_{\chi} \ll q^{1 / 2} \log q$. But for prime $q$, Vinogradov [24] proved the better bound

$$
n_{\chi} \ll q^{1 /(2 \sqrt{e})}(\log q)^{2}
$$

by combining a simple argument with (1.1). He also conjectured that $n_{\chi} \ll_{\varepsilon} q^{\varepsilon}$ for all integers $q \geq 2$ and any $\varepsilon>0$. Under the Generalized Riemann Hypothesis (GRH), Linnik [18] settled this conjecture, and later Ankeny [1] gave a sharper estimate

$$
n_{\chi} \ll(\log q)^{2}
$$

(still assuming GRH). Burgess ([3], [4], [5]) wrote a series of important papers on sharpening (1.1). His well known estimate on character sums is as follows: For any $\varepsilon>0$, there is $\delta(\varepsilon)>0$ such that

$$
\left|\sum_{n \leq x} \chi(n)\right| \ll_{\varepsilon} x q^{-\delta(\varepsilon)}
$$

provided $x \geq q^{1 / 3+\varepsilon}$. The last condition can be improved to $x \geq q^{1 / 4+\varepsilon}$ if $q$ is cubefree. When $q$ is prime, he deduced, via (1.4) and Vinogradov's argument,

$$
n_{\chi} \ll_{\varepsilon} q^{1 /(4 \sqrt{e})+\varepsilon} .
$$

Since Burgess' estimate (1.4) on character sums holds for composite modulus, one expects a bound analogous to (1.5) for $n_{\chi}$ in general cases, but this seems not available in literature. Our first result is to propose such a generalisation, by modifying Vinogradov's argument.

2000 Mathematics Subject Classification: 11K31

Key words and phrases: Special sequences 
Theorem 1. Let $\varepsilon$ be an arbitrarily small positive number. For all integers $q \geq 2$ and $\chi$ non principal characters $(\bmod q)$, we have

$$
n_{\chi} \ll_{\varepsilon} \begin{cases}q^{1 /(4 \sqrt{e})+\varepsilon} & \text { if } q \text { is cubefree, } \\ q^{1 /(3 \sqrt{e})+\varepsilon} & \text { otherwise. }\end{cases}
$$

The proof of Theorem 1 will be given in the Section 2 .

Let us now focus on real primitive characters. Denote $\mathcal{D}$ (resp. $\mathcal{D}(Q)$ ) to be the set of fundamental discriminants $d$ (resp. with $|d| \leq Q$ ), that is, the set of non-zero integers $d$ which are products of coprime factors of the form $-4,8,-8, p^{\prime}$ where $p^{\prime}:=(-1)^{(p-1) / 2} p$ ( $p$ odd prime). Also, we write $\mathcal{K}$ (resp. $\mathcal{K}(Q)$ ) for the set of real primitive characters (resp. with modulus $q \leq Q$ ). Then there is a bijection between $\mathcal{D}$ and $\mathcal{K}$ given by

$$
d \mapsto \chi_{d}(\cdot)=\left(\frac{d}{\cdot}\right)_{K}
$$

where $\left(\frac{d}{.}\right)_{K}$ is the Kronecker symbol. Note that the modulus of $\chi_{d}$ equals $|d|$ and

$$
|\mathcal{D}(Q)|=|\mathcal{K}(Q)|=\frac{6}{\pi^{2}} Q+O\left(Q^{1 / 2}\right) .
$$

In the opposite direction of (1.2), Frilender [12], Salié [23] and Chowla \& Turán (see [10]) independently shew that there are infinitely many primes $p$ for which

$$
n_{\chi_{p^{\prime}}} \gg \log p,
$$

or in other words, $n_{\chi_{p^{\prime}}}=\Omega(\log p)$. Under GRH, Montgomery [20] gave a stronger result $n_{\chi_{p^{\prime}}}=\Omega\left(\log p \log _{2} p\right)$, where $\log _{k}$ denotes the $k$-fold iterated logarithm. Without any assumption Graham \& Ringrose [14] obtained $n_{\chi_{p^{\prime}}}=\Omega\left(\log p \log _{3} p\right)$. In view of these results, it is natural to wonder what is the size of the majority of $n_{\chi_{p^{\prime}}}$, or more generally $n_{\chi_{d}}$. Indeed the density of large $n_{\chi_{p^{\prime}}}$ 's like those in (1.7) or bigger is low, which can be seen from an Erdős' result [11],

$$
\lim _{x \rightarrow \infty} \frac{1}{\pi(x)} \sum_{p \leq x} n_{\chi_{p^{\prime}}}=\text { constant }
$$

where $\pi(x)$ denotes the number of primes up to $x$. This result is extended and refined by Elliott in [7] and [8]. Using (1.8) or its refinement in [7], it follows, for any fixed constant $\delta>0$, we see

$$
\sum_{p \leq x, n_{\chi_{p^{\prime}}} \geq \delta \log p} 1 \ll_{\delta} \frac{x}{(\log x)^{2}} .
$$

In [6], Duke \& Kowalski indicated: Let $\alpha>1$ be given. Denote by $N(Q, \alpha)$ the number of primitive characters $\chi$ (not necessarily real) of modulus $q \leq Q$ such that $\chi(n)=1$ for all $n \leq(\log Q)^{\alpha}$ and $(n, q)=1$. Then one has

$$
N(Q, \alpha) \ll_{\varepsilon} Q^{2 / \alpha+\varepsilon}
$$

for all $\varepsilon>0$. This follows that

$$
\left|\left\{|d| \leq Q: n_{\chi_{d}} \geq(\log Q)^{\alpha}\right\}\right| \ll_{\varepsilon} Q^{2 / \alpha+\varepsilon} .
$$


However, in view of (1.6) this result is non-trivial only when $\alpha>2$ and it tells that $n_{\chi_{d}} \geq$ $(\log |d|)^{2+\varepsilon}$ for almost all fundamental discriminants $d$. Very recently Baier [2] improved $2+\varepsilon$ to $1+\varepsilon$ by using the large sieve inequality of Heath-Brown [15] for real primitive characters. Still it is unable to cover the case $\alpha=1$ or to provide information on the sparsity of the primes $p$ with $n_{\chi_{p^{\prime}}} \gg \log p$ as in (1.9).

Our second result is to supplement the case $\alpha=1$, using the large sieve inequality of ElliottMontgomery-Vaughan (see [9] and [21]). We obtain an almost all result, which is strong enough to yield a more tight estimate on the low density of exceptional non-residues than in (1.9).

Theorem 2. For $2 \leq P \leq Q$, define

$$
\mathcal{E}(Q, P):=\left\{d \in \mathcal{D}(Q): \chi_{d}(p)=1 \text { for } P<p \leq 2 P \text { and } p \nmid|d|\right\} .
$$

Then there are two absolute positive constants $C$ and $c$ such that

$$
|\mathcal{E}(Q, P)| \ll Q e^{-c(\log Q) / \log _{2} Q}
$$

holds uniformly for $Q \geq 10$ and $C \log Q \leq P \leq(\log Q)^{2}$. In particular we have

$$
n_{\chi_{d}} \ll \log |d|
$$

for all but except $O\left(Q e^{-c(\log Q) / \log _{2} Q}\right)$ characters $\chi_{d} \in \mathcal{K}(Q)$.

Sections 3 and e are devoted to the proof of Theorem 2.

The next Theorem 3 (essentially due to Graham \& Ringrose [14]) shows that the upper bound for exceptional real primitive characters set is optimal. Graham \& Ringrose considered a problem of the quasi-random graths (Paley graths) which leads to study the lower bound for the sum of the right-hand side of (6.5) below. This will also be the essential part of our proof of Theorem 3. We shall provide the salient points along the line of arguments in [14] to prove Theorem 3, see Sections 5 and 6 .

Theorem 3. For any fixed constant $\delta>0$, there are a sequence of positive real numbers $\left\{Q_{n}\right\}_{n=1}^{\infty}$ with $Q_{n} \rightarrow \infty$ and a positive constant $c$ such that

$$
\sum_{\substack{Q_{n}^{1 / 2}<p \leq Q_{n} \\ n_{\chi_{p}} \geq \delta \log p}} 1 \gg_{\delta} Q_{n} e^{-c\left(\log Q_{n}\right) / \log _{2} Q_{n}} .
$$

Further if we assume that both $\mathbf{L}_{1}\left(s, P_{y}\right)$ and $\mathbf{L}_{4}\left(s, P_{y}\right)$ defined in (5.3) below have no exceptional zeros in the region (5.4), then (1.12) holds for all $Q \geq 10$.

Finally we consider the least quadratic non-residue problem in Pajtechii-S̆apiro's sequence $\left\{\left[n^{c}\right]\right\}_{n=1}^{\infty}$, where $c>1$ is a constant and $[t]$ denotes the integral part of $t \in \mathbb{R}$. Denote by $n_{\chi_{p^{\prime}}, c}$ the least positive integer $n$ such that $\left[n^{c}\right]$ is a quadratic non-residue $(\bmod p)$. Garaev [13] proved that for $1<c<\frac{12}{11}$ and any $\varepsilon>0$, one has

$$
n_{\chi_{p^{\prime}}, c} \ll_{c, \varepsilon} p^{3 /(8(3-2 c) \sqrt{e})+\varepsilon}
$$

for all primes $p$. He pointed out also that by the method of exponential pairs the range of $c$ and the exponent of $p$ can be improved to $1<c<\frac{12}{11}+0.00257 \cdots$ and $1 /\left(8\left(1-\theta_{2} c\right) \sqrt{e}\right)$, respectively, where $\theta_{2}=0.66451 \cdots$. Here we propose a further improvement by applying a recent result of Robert \& Sargos [22], and give an almost result based on Theorem 2. 
Theorem 4. Let $1<c<\frac{32}{29}$. Then for all primes $p$ and any $\varepsilon>0$, we have

$$
n_{\chi_{p^{\prime}, c}} \ll_{c, \varepsilon} p^{9 /((64-40 c) \sqrt{e})+\varepsilon} .
$$

For all but except $O\left(Q e^{-c(\log Q) / \log _{2} Q}\right)$ primes $p$ with $p \leq Q$, we have

$$
n_{\chi_{p^{\prime}}, c} \ll_{c, \varepsilon}(\log p)^{9 /(16-10 c)+\varepsilon} .
$$

We prove Theorem 4 in Section 7.

Our range of $c$ is larger than $\frac{12}{11}+0.00257 \cdots\left(\frac{32}{29}=\frac{12}{11}+0.01253 \cdots\right)$ and our exponent is definitely better than $(1.13)$ but is smaller than $1 /\left(8\left(1-\theta_{2} c\right) \sqrt{e}\right)$ only when $c>1 /\left(9 \theta_{2}-5\right)=$ $1.019794 \cdots$. It is possible to give a slightly better result with Huxley's estimates for exponential sums $[16, \S 18.5]$. We can also generalize Theorem 4 to composite modulus $|d|$ as in Theorem 1 , but with smaller range of $c$ and larger exponent of $|d|$.

\section{$\S 2$. Vinogradov's argument and proof of Theorem 1}

Without loss of generality we assume $n_{\chi} \geq q^{1 /(4 \sqrt{e})}$ (otherwise there is nothing to prove). Let $x$ be a number specified later but satisfy

$$
q>x \geq \begin{cases}q^{1 / 4+\varepsilon} & \text { if } q \text { is cubefree, } \\ q^{1 / 3+\varepsilon} & \text { otherwise. }\end{cases}
$$

By Burgess' well known estimate (1.4) on character sums, for any $\varepsilon>0$ there are two positive constants $C_{\varepsilon}$ and $\delta(\varepsilon)>0$ such that

$$
\begin{aligned}
C_{\varepsilon} x q^{-\delta(\varepsilon)} & \geq\left|\sum_{n \leq x} \chi(n)\right| \\
& \geq \sum_{\substack{n \leq x \\
(n, q)=1}} 1-2 \sum_{\substack{n \leq x \\
(n, q)=1, \chi(n) \neq 1}} 1 \\
& \geq \sum_{\substack{n \leq x \\
(n, q)=1}} 1-2 \sum_{n_{\chi}<p \leq x} \sum_{\substack{m \leq x / p \\
(m, q)=1}} 1 .
\end{aligned}
$$

As usual we denote by $\varphi(n)$ the Euler function, $\mu(n)$ the Möbius function and $\omega(n)$ the number of distinct prime factors of $n$. With the Möbius inversion formula, we have, for some $|\theta| \leq 1$,

$$
\sum_{\substack{n \leq x \\(n, q)=1}} 1=\sum_{d \mid q} \mu(d) \sum_{m \leq x / d} 1=\frac{\varphi(q)}{q} x+\theta 2^{\omega(q)} .
$$

To estimate the last double sum on the right-hand side of $(2.1)$, we divide the sum over $p$ into two parts according as $n_{\chi}<p \leq x / 2^{\omega(q)}$ or $x / 2^{\omega(q)}<p \leq x$. By (2.2), the first part contributes at most

$$
\begin{aligned}
& \sum_{n_{\chi}<p \leq x / 2^{\omega(q)}}\left(\frac{\varphi(q)}{q} \frac{x}{p}+2^{\omega(q)}\right) \\
\leq & \frac{\varphi(q)}{q} x\left\{\log \left(\frac{\log x}{\log n_{\chi}}\right)+O\left(e^{-\sqrt{\log n_{\chi}}}\right)\right\}+\frac{(1+\varepsilon) x}{\log \left(x 2^{-\omega(q)}\right)} \\
\leq & \frac{\varphi(q)}{q} x \log \left(\frac{\log x}{\log n_{\chi}}\right)+(1+2 \varepsilon) \frac{x}{\log x} .
\end{aligned}
$$


Note that $2^{\omega(q)} \ll x^{\varepsilon}$ and $n_{\chi} \geq q^{1 /(4 \sqrt{e})}$. For the second part, we interchange the summations and apply the Rankin trick,

$$
\begin{aligned}
\sum_{x / 2^{\omega(q)}<p \leq x} \sum_{\substack{m \leq x / p \\
(m, q)=1}} 1 & \sum_{\substack{1 \leq m \leq 2^{\omega(q)} \\
(m, q)=1}} \sum_{p \leq x / m} 1 \\
& \ll \frac{x}{\log x} \sum_{\substack{1 \leq m \leq 2^{\omega(q)} \\
(m, q)=1}} \frac{1}{m} \\
& \leq \frac{x}{\log x} \prod_{\substack{p \leq 2^{\omega(q)} \\
(p, q)=1}}\left(1-\frac{1}{p}\right)^{-1} \\
& =\frac{\varphi(q)}{q} \frac{x}{\log x} \prod_{p>2^{\omega(q)}}\left(1-\frac{1}{p}\right)^{-1} \times \prod_{p \leq 2^{\omega(q)}}\left(1-\frac{1}{p}\right)^{-1} .
\end{aligned}
$$

In virtue of the simple estimates

$$
\begin{aligned}
& \prod_{\substack{p>2^{\omega(q)} \\
p \mid q}}\left(1-\frac{1}{p}\right)^{-1} \ll \exp \left\{\sum_{\substack{p>2^{\omega(q)} \\
p \mid q}} \frac{1}{p}\right\} \ll \exp \left\{\frac{\omega(q)}{2^{\omega(q)}}\right\} \ll 1, \\
& \prod_{p \leq 2^{\omega(q)}}\left(1-\frac{1}{p}\right)^{-1} \ll \exp \left\{\sum_{p \leq 2^{\omega(q)}} \frac{1}{p}\right\} \ll \omega(q),
\end{aligned}
$$

it follows immediately that

$$
\sum_{x / 2^{\omega(q)}<p \leq x} \sum_{\substack{m \leq x / p \\(m, q)=1}} 1 \ll \frac{\varphi(q)}{q} x \frac{\omega(q)}{\log x} .
$$

Inserting (2.2), (2.3) and (2.4) into (2.1), we conclude

$$
C_{\varepsilon} x q^{-\delta(\varepsilon)} \geq \frac{\varphi(q)}{q} x\left\{1-2 \log \left(\frac{\log x}{\log n_{\chi}}\right)\right\}-2^{\omega(q)}-(1+2 \varepsilon) \frac{x}{\log x}-C_{\varepsilon} \frac{\varphi(q)}{q} x \frac{\omega(q)}{\log x} .
$$

From this we deduce that

$$
\begin{aligned}
\log \left(\frac{\log x}{\log n_{\chi}}\right) & \geq \frac{1}{2}-\frac{C_{\varepsilon}}{2} \frac{q^{1-\delta(\varepsilon)}}{\varphi(q)}-\frac{(1 / 2+\varepsilon) q}{\varphi(q) \log x}-\frac{C_{\varepsilon}}{2} \frac{\omega(q)}{\log x} \\
& \geq \frac{1}{2}-C_{\varepsilon}\left(\frac{q}{\varphi(q) \log x}+\frac{\omega(q)}{\log x}\right)
\end{aligned}
$$

provided $q \geq q_{0}(\varepsilon)$. Since $q / \varphi(q) \log x+\omega(q) / \log x \ll\left(\log _{2} q\right)^{-1}$, the preceeding inequality implies

$$
n_{\chi} \ll x^{1 / \sqrt{e}} \exp \left\{O\left(\frac{q}{\varphi(q)}+\omega(q)\right)\right\}
$$

which gives the required result, by taking

$$
x= \begin{cases}q^{1 / 4+\varepsilon} & \text { if } q \text { is cubefree } \\ q^{1 / 3+\varepsilon} & \text { otherwise }\end{cases}
$$

This completes the proof of Theorem 1. 


\section{$\S$ 3. A large sieve inequality of Montgomery-Vaughan}

Our key tool for proving Theorem 2 is a large sieve inequality of Montgomery \& Vaughan in [21, page 1050] following from [21, Lemma 2]. Here we state a slightly refined version (see Lemma 1 below). Their original statement absorbs the factors $(6 / \log P)^{j}$ and $\left\{6 /(\log P)^{2}\right\}^{j}$ in the implied constant. We reproduce here their proof with a minuscule modification.

Lemma 1. We have

$$
\sum_{d \in \mathcal{D}(Q)}\left|\sum_{P<p \leq 2 P} \frac{\chi_{d}(p)}{p}\right|^{2 j} \ll Q\left(\frac{6 j}{P \log P}\right)^{j}+\left(\frac{6 P}{(\log P)^{2}}\right)^{j}
$$

uniformly for $2 \leq P \leq Q$ and $j \geq 1$. The implied constant is absolute.

Proof. Since $\chi_{d}(n)$ is completely multiplicative on $n$, we can write

$$
\left(\sum_{P<p \leq 2 P} \frac{\chi_{d}(p)}{p}\right)^{j}=\sum_{P^{j}<m \leq(2 P)^{j}} \frac{a_{j}(m)}{m} \chi_{d}(m)
$$

where

$$
a_{j}(m):=\left|\left\{\left(p_{1}, \ldots, p_{j}\right): p_{1} \cdots p_{j}=m, P<p_{i} \leq 2 P\right\}\right| .
$$

By Lemma 2 of [21] with the choice of parameters

$$
X=P^{j}, \quad Y=(2 P)^{j} \quad \text { and } \quad a_{m}=a_{j}(m) / m,
$$

it follows that as $a_{j}\left(m_{1}\right) a_{j}\left(m_{2}\right) \leq a_{2 j}\left(n^{2}\right)$ for $n^{2}=m_{1} m_{2}$,

$$
\sum_{d \in \mathcal{D}(Q)}\left|\sum_{P<p \leq 2 P} \frac{\chi_{d}(p)}{p}\right|^{2 j} \ll Q \sum_{P^{j}<n \leq(2 P)^{j}} \frac{a_{2 j}\left(n^{2}\right)}{n^{2}}+\left(\sum_{P<p \leq 2 P} \frac{1}{p^{1 / 2}}\right)^{2 j} .
$$

Writing $n=p_{1}^{\nu_{1}} \cdots p_{i}^{\nu_{i}}$ with $\nu_{1}+\cdots+\nu_{i}=j$, we have

$$
\begin{aligned}
a_{2 j}\left(n^{2}\right) & =\frac{(2 j) !}{\left(2 \nu_{1}\right) ! \cdots\left(2 \nu_{i}\right) !} \\
& =\frac{(2 j) !}{j !} \frac{\nu_{1} !}{\left(2 \nu_{1}\right) !} \cdots \frac{\nu_{i} !}{\left(2 \nu_{i}\right) !} a_{j}(n) .
\end{aligned}
$$

From this, it is easy to see $a_{2 j}\left(n^{2}\right) \leq j^{j} a_{j}(n)$, and thus

$$
\begin{aligned}
\sum_{P^{j}<n \leq(2 P)^{j}} \frac{a_{2 j}\left(n^{2}\right)}{n^{2}} & \leq j^{j} \sum_{P^{j}<n \leq(2 P)^{j}} \frac{a_{j}(n)}{n^{2}} \\
& =\left(j \sum_{P<p \leq 2 P} \frac{1}{p^{2}}\right)^{j} \\
& \leq\left(\frac{6 j}{P \log P}\right)^{j} .
\end{aligned}
$$

Inserting it into (3.2) and using the estimate

$$
\sum_{P<p \leq 2 P} \frac{1}{p^{1 / 2}} \leq \frac{6 P^{1 / 2}}{\log P}
$$

we obtain the required result (3.1). 


\section{$\S$ 4. Proof of Theorem 2}

Define

$$
\mathcal{E}^{*}(Q, P):=\left\{d \in \mathcal{D}(Q): Q^{1 / 2} \leq|d| \leq Q \text { and } \chi_{d}(p)=1 \quad(P<p \leq 2 P, p \nmid|d|)\right\}
$$

Let $C \log Q \leq P \leq(\log Q)^{2}$. For $d \in \mathcal{E}^{*}(Q, P)$, we invoke the prime number theorem to deduce

$$
\begin{aligned}
\sum_{P<p \leq 2 P} \frac{\chi_{d}(p)}{p} & =\sum_{P<p \leq 2 P} \frac{1}{p}-\sum_{P<p \leq 2 P, p|| d \mid} \frac{1}{p} \\
& \geq \frac{\log 2+o(1)}{\log P}-\frac{\{1+o(1)\} \log Q}{P \log _{2} Q} \\
& \geq \frac{\log 2-2 / C+o(1)}{\log P} \\
& >\frac{1}{2 \log P},
\end{aligned}
$$

provided $C$ is sufficiently large. It is apparent from (3.1) that

$$
\begin{aligned}
\frac{\left|\mathcal{E}^{*}(Q, P)\right|}{(2 \log P)^{2 j}} & \leq \sum_{d \in \mathcal{D}(Q)}\left|\sum_{P<p \leq 2 P} \frac{\chi_{d}(p)}{p}\right|^{2 j} \\
& \ll Q\left(\frac{6 j}{P \log P}\right)^{j}+\left(\frac{6 P}{(\log P)^{2}}\right)^{j} .
\end{aligned}
$$

Hence we obtain

$$
\left|\mathcal{E}^{*}(Q, P)\right| \ll Q(12 j \log P / P)^{j}+(12 P)^{j}
$$

uniformly for $C \log Q \leq P \leq(\log Q)^{2}$ and $j \geq 1$. Taking

$$
j=\left[\frac{\log Q}{48 \log P}\right]+1,
$$

a simple calculation shows that

$$
\left|\mathcal{E}^{*}(Q, P)\right| \ll Q e^{-c(\log Q) / \log _{2} Q}
$$

with $c=(\log 2) / 48$. This implies (1.10).

Finally let

$$
\mathcal{E}^{*}(Q):=\left\{d \in \mathcal{D}(Q): d \leq Q^{1 / 2}\right\} \cup \mathcal{E}^{*}(Q, C \log Q)
$$

Then by (1.10), we have

$$
\left|\mathcal{E}^{*}(Q)\right| \ll Q e^{-c(\log Q) / \log _{2} Q}
$$

and for any $d \in \mathcal{D}(Q) \backslash \mathcal{E}^{*}(Q)$ there is a prime number $p \asymp \log Q \asymp \log |d|$ such that $\chi_{d}(p) \neq 1$, which implies (1.11). The proof is complete. 


\section{$\S$ 5. Graham-Ringrose's method}

In this section, we shall state and extend the main results of ([14], Theorems 2, 3 and 4) for our purposes. For characters of certain moduli, Graham \& Ringrose [14] obtained a wide zero-free region and good zero density estimates for the corresponding Dirichlet $L$-functions. The main ingredient of their method is an $q$-analogue of van der Corput's result, which can be stated as follows: Suppose that $q=2^{\nu} r$, where $0 \leq \nu \leq 3$ and $r$ is an odd squarefree integer, and that $\chi$ is a non-prinicipal charater $\bmod q$. Let $p$ be the largest prime factor of $q$. Suppose that $k$ is a non-negative integer, and $K=2^{k}$. Finally, assume that $N \leq M$. Then

$$
\sum_{M<n \leq M+N} \chi(n) \ll M^{1-\frac{k+3}{8 K-2}} p^{\frac{k^{2}+3 k+4}{32 K-8}} q^{\frac{1}{8 K-2}} d(q)^{\frac{32 k^{2}+11 k+8}{16 K-4}}(\log q)^{\frac{k+3}{8 K-2}} \sigma_{-1}(q),
$$

where $\sigma_{a}(q):=\sum_{d \mid q} d^{a}$ and $d(q):=\sigma_{0}(q)$. The implied constant is absolute.

Recall that for any odd prime $p$,

$$
\chi_{8}(p)=\left(\frac{2}{p}\right), \quad \chi_{q^{\prime}}(p)=\left(\frac{q}{p}\right)_{K}=\left(\frac{q}{p}\right) \quad\left(q \text { odd prime, } q^{\prime}:=(-1)^{(q-1) / 2} q\right)
$$

by definition. For squarefree $m \geq 2$, the character $\chi_{m}:=\prod_{p \mid m} \chi_{p^{\prime}}$ for odd $m$ or $\chi_{m}:=\chi_{8} \chi_{m^{\prime}}$ for $m=2 m^{\prime}$ is a real primitive of modulus $m$ or $4 m$, respectively. By convention, we set $\chi_{1} \equiv 1$. Moreover, if $\chi_{4}$ is the real primitive character $\bmod 4$, i.e. $\chi_{4}(n)=(-1)^{(n-1) / 2}$ for odd $n$, then $\chi_{4 m}:=\chi_{4} \chi_{m}$ is also a real primitive character of modulus $4 m$.

Let

$$
P_{y}:=\prod_{p \leq y} p=e^{\{1+o(1)\} y} \quad(y \rightarrow \infty)
$$

and define for $\ell=1$ or 4 ,

$$
\mathbf{L}_{\ell}\left(s, P_{y}\right):=\prod_{m \mid P_{y}} L\left(s, \chi_{\ell m}\right),
$$

where $L\left(s, \chi_{\ell m}\right)$ is the Dirichlet $L$-function associated to $\chi_{\ell m}$. Denote by $N_{\ell}(\alpha)$ the number of zeros of $\mathbf{L}_{\ell}\left(s, P_{y}\right)$ in the rectangle

$$
\alpha \leq \sigma \leq 1 \quad \text { and } \quad|\tau| \leq \log P_{y} .
$$

Here and in the sequel we implicitly define the real numbers $\sigma$ and $\tau$ by the relation $s=\sigma+i \tau$.

The next lemmas 2, 3 and 4 are trivial extensions of Theorems 2,3 and 4 of [14], respectively.

Lemma 2. Let $y \geq 100$. Then there is an absolute positive constant $C_{1}$ such that the $L$-function $\prod_{\ell=1,4} \mathbf{L}_{\ell}\left(s, P_{y}\right)$ has at most one zero in the region

$$
\sigma \geq 1-\frac{C_{1}\left(\log _{2} P_{y}\right)^{1 / 2}}{\log P_{y}} \quad \text { and } \quad|\tau| \leq \log P_{y} .
$$

The exceptional zero, if exists, is real.

Proof. As the crucial estimate (5.1) holds for all non-principal primitive characters of modulus $q=2^{\nu} r \geq 2$ with $0 \leq \nu \leq 3$ and $r$ being odd squarefree. Consider the case $\nu=0$ or 3 , and $\nu=2$ or 3 , respectively. We see that (5.1) applies to $\chi_{m}$ and $\chi_{4 m}$ for any $m \mid P_{y}$. It follows that $[14$, Lemma 6.1] is valid for these characters. Proceeding with the same argument, we have [14, Lemma 6.2] for our $L$-function $\prod_{\ell=1,4} \mathbf{L}_{\ell}\left(s, P_{y}\right)$ in place of $\mathbf{L}\left(s, P_{y}\right)$ there. Then the same proof of $[14$, Theorem 2] will give the desired result. (Note that the value of $\phi$ suffers a negligible change when $P_{y}$ is replaced by $4 P_{y}$ or $8 P_{y}$.) The exceptional zero must be real, for otherwise, its conjugate is another zero in the specified region. 
Lemma 3. Let $C_{1}$ be as in Lemma 2. There is a sequence of positive real numbers $\left\{y_{n}\right\}_{n=1}^{\infty}$ with $y_{n} \rightarrow \infty$ such that both $\mathbf{L}_{1}\left(s, P_{y_{n}}\right)$ and $\mathbf{L}_{4}\left(s, P_{y_{n}}\right)$ have no zeros in the region

$$
\sigma \geq 1-\eta\left(y_{n}\right) \quad \text { and } \quad|\tau| \leq \log P_{y_{n}}
$$

where

$$
\eta(y):=\frac{C_{1}\left(\log _{2} P_{y}\right)^{1 / 2}}{2 \log P_{y}} .
$$

Proof. Similar to [14, Theorem 3], our proof is also based on an interesting argument attributed to Maier [19]. Suppose that for some $y$, the product $\mathbf{L}_{1}\left(s, P_{y}\right) \mathbf{L}_{4}\left(s, P_{y}\right)$ has an exceptional zero in the region (5.4). That is, it has a real zero $\beta>1-2 \eta(y)$. In view of (5.2), we can take $y_{n} \geq y$ such that

$$
\eta\left(y_{n}\right)<1-\beta<2 \eta\left(y_{n}\right) .
$$

By Lemma $2, \beta$ is the only exceptional zero of $\prod_{\ell=1,4} \mathbf{L}_{\ell}\left(s, P_{y_{n}}\right)$ in the region

$$
\sigma>1-2 \eta\left(y_{n}\right) \quad \text { and } \quad|\tau| \leq \log P_{y_{n}} .
$$

Together with the first inequality in (5.6), this forces $\prod_{\ell=1,4} \mathbf{L}_{\ell}\left(s, P_{y_{n}}\right)$ to have no zero in the region (5.5). It follows that we can find a sequence of positive real numbers $\left\{y_{n}\right\}_{n=1}^{\infty}$ with $y_{n} \rightarrow \infty$ such that both $\mathbf{L}_{1}\left(s, P_{y_{n}}\right)$ and $\mathbf{L}_{4}\left(s, P_{y_{n}}\right)$ have no zero in this region.

Lemma 4. Let $\ell=1$ or 4 and $y \geq 100$. Then there is an absolute constant $C_{2}$ such that

$$
N_{\ell}(\alpha) \ll \begin{cases}\exp \left\{\frac{C_{2}(1-\alpha) \log P_{y}}{\left.\sqrt{\log _{2} P_{y}}+\frac{\log _{3} P_{y}}{2}\right\}}\right. & \text { if } \alpha \geq 1-\eta_{1}(y), \\ \exp \left\{\frac{C_{2}(1-\alpha) \log P_{y}}{\log (1 /(1-\alpha))}\right\} & \text { if } \alpha<1-\eta_{1}(y),\end{cases}
$$

where

$$
k_{0}(y):=\left[\left(\log _{2} P_{y}\right)^{1 / 2}\right] \quad \text { and } \quad \eta_{1}(y):=\frac{k_{0}(y)}{2\left(2^{k_{0}(y)}-2\right)}
$$

Proof. The case of $\ell=1$ has been done in [14, Sections 7 and 8] and $N_{4}(\alpha)$ can be treated in the same way by applying (5.1) to our $\chi_{4 m}$.

\section{$\S$ 6. Proof of Theorem 3}

In this section, we denote by $p$ and $q$ prime numbers. Define

$$
\mathbb{P}_{y}:=\left\{p: p \equiv 1(\bmod 4) \text { and } \chi_{p}(q)=1 \text { for all } q \leq y\right\}
$$

Clearly we have $n_{\chi_{p}}>y$ for any $p \in \mathbb{P}_{y}$. We shall first show that the set $\mathbb{P}_{y}$ is not too small for suitable $y$. 
Proposition. Let $\delta>0$ be a fixed small constant and $y(x)$ be an strictly increasing function defined on $[120, \infty)$ satisfying

$$
(\log x) e^{-\delta\left(\log _{2} x\right)^{1 / 2}} \leq y(x) \leq \delta(\log x) \log _{3} x .
$$

Then there are a positive constant $c=c(\delta)$ and a sequence of positive real numbers $\left\{x_{n}\right\}_{n=1}^{\infty}$ with $x_{n} \rightarrow \infty$ such that

$$
\sum_{\substack{x_{n}^{1 / 2}<p \leq x_{n} \log x_{n} \\ p \in \mathbb{P}_{y\left(x_{n}\right)}}} 1 \gg x_{n} e^{-c y\left(x_{n}\right) / \log y\left(x_{n}\right)} .
$$

Further if we assume that both $\mathbf{L}_{1}\left(s, P_{y}\right)$ and $\mathbf{L}_{4}\left(s, P_{y}\right)$ have no zeros in the region (5.4) for all $y \geq 100$, then there is a positive constant $c$ such that for all $x \geq 100$ we have

$$
\sum_{\substack{x^{1 / 2}<p \leq x \\ p \in \mathbb{P}}} 1 \gg x e^{-c y(x) / \log y(x)} .
$$

Proof. First let $10 \leq y \leq x^{1 / 2}$. As usual, $\pi(y)$ denotes the number of prime numbers $\leq y$. Clearly we have

$$
2^{-\pi(y)-1}\left(1+\chi_{4}(p)\right) \prod_{q \leq y}\left(1+\chi_{p}(q)\right)= \begin{cases}1 & \text { if } p \in \mathbb{P}_{y} \\ 0 & \text { if } p \notin \mathbb{P}_{y}\end{cases}
$$

When $p$ and $q$ are odd primes with $p \equiv 1(\bmod 4)$, i.e. $\chi_{4}(p)=1$, we infer by quadratic reciprocity law that

$$
\chi_{p}(q)=\left(\frac{p}{q}\right)=\left(\frac{q}{p}\right)=\chi_{q^{\prime}}(p) \quad\left(q^{\prime}:=(-1)^{(q-1) / 2} q\right) .
$$

Note also for odd prime $p$,

$$
\chi_{p}(2)=\left(\frac{p}{2}\right)_{K}=\left(\frac{2}{p}\right)=\chi_{8}(p) .
$$

Thus we can replace $\chi_{p}(q)$ by $\left(\frac{q}{p}\right)$ in (6.4) to write

$$
\sum_{\substack{x^{1 / 2}<p \leq x \log x \\ p \in \mathbb{P}_{y}}} 1=\frac{1}{2^{\pi(y)+1}} \sum_{x^{1 / 2}<p \leq x \log x}\left(1+\chi_{4}(p)\right) \prod_{q \leq y}\left(1+\left(\frac{q}{p}\right)\right) .
$$

It is more convenient to introduce the weight factor $(\log p)\left(e^{-p /(2 x)}-e^{-p / x}\right)$ to the summands,

$$
\begin{aligned}
\sum_{\substack{x^{1 / 2}<p \leq x \log x \\
p \in \mathbb{P}_{y}}} 1 \geq \frac{1}{2^{\pi(y)+2} \log x} \sum_{x^{1 / 2}<p \leq x \log x}(\log p)\left(e^{-p /(2 x)}-e^{-p / x}\right) \times \\
\quad \times\left(1+\chi_{4}(p)\right) \prod_{q \leq y}\left(1+\left(\frac{q}{p}\right)\right) .
\end{aligned}
$$

We want to relax the range of the sum over $p$. To this end, we observe that by the prime number theorem and integration by parts,

$$
\begin{aligned}
& \frac{1}{2^{\pi(y)} \log x} \sum_{x \log x<p \leq x^{2}}(\log p)\left(e^{-p /(2 x)}-e^{-p / x}\right)\left(1+\chi_{4}(p)\right) \prod_{q \leq y}\left(1+\left(\frac{q}{p}\right)\right) \\
& \quad \ll \sum_{x \log x<p \leq x^{2}}\left(e^{-p /(2 x)}-e^{-p / x}\right) \\
& \ll x^{1 / 2} / \log x .
\end{aligned}
$$


Combining this with the preceeding inequality, we obtain

$$
\sum_{\substack{x^{1 / 2}<p \leq x \log x \\ p \in \mathbb{P}_{y}}} 1 \geq \frac{1}{(\log x) 2^{\pi(y)+2}} \sum_{m \mid P_{y}}\left(S_{x}(m)+S_{x}(4 m)\right)+O\left(\frac{x^{1 / 2}}{\log x}\right),
$$

where $\ell=1$ or 4 , and

$$
S_{x}(\ell m):=\sum_{x^{1 / 2}<p \leq x^{2}}(\log p)\left(e^{-p /(2 x)}-e^{-p / x}\right) \chi_{\ell m}(p) .
$$

By the Perron formula, we can write

$$
S_{x}(\ell m)=\frac{1}{2 \pi i} \int_{2-i \infty}^{2+i \infty}-\frac{L^{\prime}}{L}\left(s, \chi_{\ell m}\right)\left(2^{s}-1\right) \Gamma(s) x^{s} \mathrm{~d} s+O\left(x^{1 / 2} \log x\right) .
$$

We shift the line of integration to $\sigma=-\frac{3}{4}$. The function $\left(2^{s}-1\right) \Gamma(s) x^{s}$ has no pole in the strip $-\frac{3}{4} \leq \sigma \leq 2$ since the pole of $\Gamma(s)$ at $s=0$ is canceled by the zero of $\left(2^{s}-1\right)$. Thus the only poles of the integrand in (6.6) occur at $s=1$ if $\ell m=1$ (note that $L\left(s, \chi_{1}\right)$ is the Riemann $\zeta$-function), or at the zeros $\rho(\ell m)=\beta(\ell m)+i \gamma(\ell m)$ of $L\left(s, \chi_{\ell m}\right)$. It follows that

$$
S_{x}(\ell m)=\delta_{\ell m, 1} x-\sum_{\rho(\ell m)}\left(2^{\rho(\ell m)}-1\right) \Gamma(\rho(\ell m)) x^{\rho(\ell m)}+O\left(x^{1 / 2} \log x\right),
$$

where $\delta_{j, 1}=1$ if $j=1$ and 0 otherwise, and the sum is over all zeros with $0 \leq \beta(\ell m)<1$.

We write $N\left(T, \chi_{\ell m}\right)$ for the number of zeros of $L\left(s, \chi_{\ell m}\right)$ in the rectangle $0<\beta(\ell m)<1$ and $|\gamma| \leq T$. Then we have the classical bound

$$
N\left(T, \chi_{\ell m}\right) \ll T \log (T m),
$$

which implies, for any $\alpha \in(0,1)$,

$$
N_{\ell}(\alpha) \leq \sum_{m \mid P_{y}} N\left(\log P_{y}, \chi_{\ell m}\right) \ll 2^{\pi(y)} y^{2} .
$$

On the other hand, by means of $\left(2^{s}-1\right) \Gamma(s) x^{s} \ll x^{\sigma}|\tau| e^{-(\pi / 2)|\tau|}$, the contribution of the zeros with $|\gamma(\ell m)| \geq \log P_{y}$ to $S_{x}(\ell m)$ is $\ll 1$. Let $\varepsilon$ be an arbitrarily small positive number. The zeros with $\beta(\ell m) \leq 1-\varepsilon$ and $|\gamma(\ell m)| \leq \log P_{y}$ contribute

$$
\ll x^{1-\varepsilon} N\left(\log P_{y}, \chi_{\ell m}\right) \ll x^{1-\varepsilon}\left(\log P_{y}\right)^{2} \ll x^{1-\varepsilon} y^{2} .
$$

Combining these with (6.5), we conclude

$$
\sum_{\substack{x^{1 / 2}<p \leq x \log x \\ p \in \mathbb{P} y}} 1 \geq \frac{x}{(\log x) 2^{\pi(y)+2}}+O\left(x^{1-\varepsilon} 2^{\pi(y)} y^{2}+\frac{T_{1}(x, y)+T_{4}(x, y)}{(\log x) 2^{\pi(y)}}\right)
$$

uniformly for $x \geq 10$ and $1 \leq y \leq x^{1 / 2}$, where

$$
\begin{aligned}
T_{\ell}(x, y) & :=\sum_{m \mid P_{y}} \sum_{\substack{\rho(\ell m) \\
\beta(\ell m) \geq 1-\varepsilon,|\gamma(\ell m)| \leq \log P_{y}}} x^{\beta(\ell m)} \\
& =-\int_{1-\varepsilon}^{1} x^{\alpha} \mathrm{d} N_{\ell}(\alpha) .
\end{aligned}
$$


It remains to estimate $T_{\ell}(x, y)$. From now on we take $y=y(x)$. By integration by parts and by using (6.8), we can deduce

$$
T_{\ell}(x, y) \ll x^{1-\varepsilon} 2^{\pi(y)} y^{2}+x(\log x) I_{\ell},
$$

where

$$
I_{\ell}:=\int_{0}^{\varepsilon} x^{-\beta} N_{\ell}(1-\beta) \mathrm{d} \beta .
$$

Let $\eta=\eta(y)$ and $\eta_{1}=\eta_{1}(y)$ be defined as in Lemmas 3 and 4 , respectively. Set $\eta_{2}:=$ $2 y(x) /(\log x) \log y$. It is easy to verify that $0<\eta<\eta_{1}<\eta_{2}<\varepsilon$. (The inequality $\eta_{1}<\eta_{2}$ governs the lower bound of $y(x)$ in (6.1).) Thus we can divide the interval $[0, \varepsilon]$ into four subintervals $[0, \eta],\left[\eta, \eta_{1}\right],\left[\eta_{1}, \eta_{2}\right]$ and $\left[\eta_{2}, \varepsilon\right]$, and denote by $I_{\ell, 0}, I_{\ell, 1}, I_{\ell, 2}$ and $I_{\ell, 3}$ the corresponding contribution to $I_{\ell}$. Plainly we have

$$
\frac{1}{2} \log _{3} P_{y} \leq \frac{\eta}{4} \log x, \quad \frac{C_{2} \log P_{y}}{\sqrt{\log _{2} P_{y}}} \leq \frac{1}{4} \log x, \quad \frac{C_{2} \log P_{y}}{\log \left(1 / \eta_{2}\right)} \leq \frac{1}{2} \log x, \quad \frac{y}{\log y}=\frac{\eta_{2}}{2} \log x .
$$

(The third inequality governs the upper bound of $y(x)$ in (6.1).) From Lemma 4 and (6.8), we deduce that

$$
\begin{aligned}
& I_{\ell, 1} \ll \int_{\eta}^{\eta_{1}} \exp \left\{-\beta \log x+\frac{C_{2} \beta \log P_{y}}{\sqrt{\log _{2} P_{y}}}+\frac{1}{2} \log _{3} P_{y}\right\} \mathrm{d} \beta \ll \frac{x^{-\eta / 2}}{\log x}, \\
& I_{\ell, 2} \ll \int_{\eta_{1}}^{\eta_{2}} \exp \left\{-\beta \log x+\frac{C_{2} \beta \log P_{y}}{\log (1 / \beta)}\right\} \mathrm{d} \beta \ll \frac{x^{-\eta_{1} / 2}}{\log x}, \\
& I_{\ell, 3} \ll \int_{\eta_{2}}^{\varepsilon} \exp \left\{-\beta \log x+\frac{y}{\log y}\right\} \mathrm{d} \beta \ll \frac{x^{-\eta_{2} / 2}}{\log x} .
\end{aligned}
$$

Hence, all of them satisfy

$$
I_{\ell, i}=o\left((\log x)^{-1}\right) \quad(i=1,2,3) .
$$

If we assume that both $\mathbf{L}_{1}\left(s, P_{y}\right)$ and $\mathbf{L}_{4}\left(s, P_{y}\right)$ have no zeros in the region (5.4) for all $y \geq 100$, then $I_{\ell, 0}=0$. Otherwise we use Lemma 3 to assure the existence of $\left\{y_{n}\right\}_{n=1}^{\infty}$ such that $I_{\ell, 0}=0$.

With (6.10), our conclusion is

$$
T_{\ell}\left(x_{n}, y_{n}\right)=o\left(\frac{x_{n}}{\left(\log x_{n}\right) 2^{\pi\left(y_{n}\right)}}\right) \quad(n \rightarrow \infty),
$$

or

$$
T_{\ell}(x, y)=o\left(\frac{x}{(\log x) 2^{\pi(y)}}\right) \quad(x \rightarrow \infty)
$$

under the assumption that both $\mathbf{L}_{1}\left(s, P_{y}\right)$ and $\mathbf{L}_{4}\left(s, P_{y}\right)$ have no exceptional zeros. Clearly this and (6.9) imply the required result. This completes the proof of Proposition.

Now we are ready to prove Theorem 3 .

Taking $Q_{n}=x_{n} \log x_{n}$ and $y(x)=100 \delta \log x$ in Proposition and noticing that $p \in \mathbb{P}_{y} \Rightarrow$ $n_{\chi_{p}} \geq y$, we have

$$
\sum_{\substack{\left(Q_{n} / \log Q_{n}\right)^{1 / 2}<p \leq Q_{n} \\ n_{\chi_{p}} \geq 100 \delta \log Q_{n}}} 1 \gg Q_{n} e^{-c_{1}\left(\log Q_{n}\right) / \log _{2} Q_{n}} .
$$

It implies the first assertion of Theorem 2, and the second one can be treated similarly. This concludes Theorem 2. 


\section{$\S$ 7. Proof of Theorem 4}

Let $1<c<\frac{32}{29}$ and $\varepsilon$ be an arbitrary but sufficiently small positive constant. The upshot is to show

$$
n_{\chi_{p^{\prime}}, c} \ll n_{\chi_{p^{\prime}}}^{9 /(16-10 c)+\varepsilon}
$$

whenever $n_{\chi_{p^{\prime}}} \geq N_{0}(c, \epsilon)$ for some suitably large constant $N_{0}(c, \varepsilon)$ depending only on $c$ and $\varepsilon$. Once (7.1) is established, the required results follow from Burgess' upper bound (1.5) or (1.11).

To prove (7.1), we make use of the observation that the integer $m n_{\chi_{p^{\prime}}}$ is quadratic nonresidue for any integer $m<n_{\chi_{p^{\prime}}}$. Now, we want to find a positive $M\left(<\frac{1}{2} n_{\chi_{p^{\prime}}}\right)$ as small as possible such that

$$
\left[n^{c}\right]=m n_{\chi_{p^{\prime}}}
$$

for some integers $m \in(M, 2 M]$ and $n>1$. This implies

$$
n_{\chi_{p^{\prime}, c}} \ll\left(M n_{\chi_{p^{\prime}}}\right)^{1 / c}
$$

which leads to (7.1) with a suitable estimate on $M$.

Apparently, (7.2) is equivalent to

$$
\left(m n_{\chi_{p^{\prime}}}\right)^{1 / c} \leq n<\left(m n_{\chi_{p^{\prime}}}+1\right)^{1 / c} .
$$

Denote by $\{x\}$ the fractional part of $x$. Then (7.4) holds if

$$
0<\left\{\left(m n_{\chi_{p^{\prime}}}+1\right)^{1 / c}\right\} \leq\left(2^{1 / c-2} / c\right)\left(M n_{\chi_{p^{\prime}}}\right)^{1 / c-1}=: \Delta<1 \quad(c>1),
$$

since

$$
\left(m n_{\chi_{p^{\prime}}}+1\right)^{1 / c}-\left(m n_{\chi_{p^{\prime}}}\right)^{1 / c} \geq(1 / c)\left(2 M n_{\chi_{p^{\prime}}}\right)^{1 / c-1} .
$$

Let $\delta_{\Delta}(t)$ be the periodic function of period 1 such that $\delta_{\Delta}(t)=1$ if $t \in(0, \Delta]$ and $=0$ if $t \in(\Delta, 1]$. Then (7.5) will follow from

$$
\sum_{M<m \leq 2 M} \delta_{\Delta}\left(\left(m n_{\chi_{p^{\prime}}}+1\right)^{1 / c}\right)>0 .
$$

Introducing the function $\psi(t):=\frac{1}{2}-\{t\}$, we can express

$$
\delta_{\Delta}(t)=\Delta+\psi(\Delta-t)-\psi(-t) .
$$

Thus we have

$$
\sum_{M<m \leq 2 M} \delta_{\Delta}\left(\left(m n_{\chi_{p^{\prime}}}+1\right)^{1 / c}\right)=\Delta M+R
$$

where

$$
R:=\sum_{M<m \leq 2 M}\left(\psi\left(\Delta-\left(m n_{\chi_{p^{\prime}}}+1\right)^{1 / c}\right)-\psi\left(-\left(m n_{\chi_{p^{\prime}}}+1\right)^{1 / c}\right)\right) .
$$

Consider respectively

$$
f(t)=\Delta-\left((M+t) n_{\chi_{p^{\prime}}}+1\right)^{1 / c}, \quad f(t)=-\left((M+t) n_{\chi_{p^{\prime}}}+1\right)^{1 / c} .
$$


Then the treatment of $R$ is reduced to the sum $\sum_{M<m<2 M} \psi(f(m))$, which can be handled using a recent result in [22] via third derivative of $f(t)$. Applying Theorem 2 of [22], we obtain

$$
R \ll_{c, \varepsilon}\left\{M\left(M^{1 / c-3} n_{\chi_{p^{\prime}}}^{1 / c}\right)^{3 / 19}+M^{3 / 4}+\left(M^{1 / c-3} n_{\chi_{p^{\prime}}}^{1 / c}\right)^{-1 / 3}\right\} M^{\varepsilon^{2}} .
$$

Thus (7.6) will hold provided

$$
M^{1-\varepsilon} \geq n_{\chi_{p^{\prime}}}^{(19 c-16) /(16-10 c)} .
$$

Taking $M=n_{\chi_{p^{\prime}}}^{(19 c-16) /(16-10 c)+\varepsilon}$, it follows that

$$
R \leq C_{0}(c, \varepsilon) n_{\chi_{p^{\prime}}}^{\varepsilon(10 c-16) / 19 c} M^{\varepsilon^{2}} \Delta M
$$

for $n_{\chi_{p^{\prime}}} \geq N_{1}(c, \varepsilon)$ where $C_{0}(c, \varepsilon)$ and $N_{1}(c, \varepsilon)$ are absolute constants depending only on $c$ and $\varepsilon$. The hypothesis $1<c<\frac{32}{29}$ yields that $M<\frac{1}{2} n_{\chi_{p^{\prime}}}$ for all sufficently large $n_{\chi_{p^{\prime}}}$. Furthermore, this hypothesis assures that the exponent of $n_{\chi_{p^{\prime}}}$ is negative and hence $R$ is suppressed by $\Delta M$ for all large $n_{\chi_{p^{\prime}}}$. Consequently, we derive (7.6) for $n_{\chi_{p^{\prime}}} \geq N_{2}(c, \epsilon)$, and therefore (7.1) by inserting the value of $M$ into (7.3). The proof of Theorem 4 is thus complete.

\section{References}

[1] N.C. Ankeny, The least quadratic non residue, Ann. of Math. (2) 55 (1952), 65-72.

[2] S. BAier, A remark on the least $n$ with $\chi(n) \neq 1$, Preprint.

[3] D.A. Burgess, The distribution of quadratic residues and non-residues. Mathematika 4 (1957), 106-112.

[4] D.A. Burgess, On character sums and L-series. II, Proc. London Math. Soc. (3) 131963 $524-536$.

[5] D.A. Burgess, The character sum estimate with $r=3$, J. London Math. Soc. (2) 33 (1986), 219-226.

[6] W. Duke \& E. Kowalski, A problem of Linnik for elliptic curves and mean-value estimates for automorphic representations. With an appendix by Dinakar Ramakrishnan. Invent. Math. 139 (2000), no. 1, 1-39.

[7] P.D.T.A. Elliott, A problem of Erdős concerning power residue sums. Acta Arith. 13 (1967/1968), 131-149.

[8] P.D.T.A. Elliott, The distribution of power residues and certain related results. Acta Arith. 17 (1970), 141-159.

[9] P.D.T.A. Elliotr, On the mean value of $f(p)$, Proc. London Math. Soc. (3) 21 (1970), 28-796.

[10] P. ERDős, MR 0045159 on the paper [1] by N.C. Ankeny.

[11] P. ERDős, Remarks on number theory. I. Mat. Lapok 12 1961, 10-17.

[12] V.R. Fridlender, On the least $n$-th power non-residue, Dokl. Akad. Nauk SSSR 66 (1949), 351-352.

[13] M.Z. Garaev, A note on the least quadratic non-residue of the integer-sequences, Bull. Austral. Math. Soc. 68 (2003), 1-11.

[14] S.W. Graham \& C.J. Ringrose, Lower bounds for least quadratic nonresidues. Analytic number theory, 269-309, Progr. Math., 85, Birkhäuser Boston, 1990. 
[15] D.R. Heath-Brown, A mean value estimate for real character sums, Acta Arith. 72 (1995), 235-275.

[16] M.N. Huxley, Area, lattice points, and exponential sums, London Mathematical Society Monographs. New Series, 13, Oxford Science Publications, The Clarendon Press, Oxford University Press, New York, 1996. xii+494 pp.

[17] E. Kowalski, Variants of recognition problems for modular forms, Arch. Math. (Basel) 84 (2005), no. 1, 57-70.

[18] U.V. Linnik, A remark on the least quadratic non-residue, C. R. (Doklady) Acad. Sci. URSS (N.S.) 36 (1942), 119-120.

[19] H. MAier, Chains of large gaps between consecutive primes, Adv. in Math. 39 (3) (1981), $257-269$

[20] H.L. Montgomery, Topics in multiplicative number theory, Lecture Notes in Math. 227, Springer-Verlag, New York, 1971.

[21] H.L. Montgomery \& R.C. Vaughan, Extreme values of Dirichlet $L$-functions at 1, in: Number theory in progress, Vol. 2, Zakopane-Kościelisko, 1997 (K. Györy, H. Iwaniec \& J. Urbanowicz, Eds), 1039-1052, de Gruyter, Berlin, 1999.

[22] O. Robert \& P. Sargos, A third derivative test for mean values of exponential sums with application to lattice point problems, Acta Arith. 106 (2003), 27-39.

[23] H. SAliÉ, Uber den kleinsten positiven quadratischen Nichtrest nach einer Primzahl, Math. Nachr. 3 (1949), 7-8.

[24] I.M. Vinogradov, Sur la distribution des résidus et non résidus de puissances, Permski J. Phys. Isp. Ob. -wa 1 (1918), 18-28 and 94-98.

Department of Mathematics, The University of Hong Kong, Pokfulam Road, HONG KONG

E-mail: yklau@maths.hku.hk

Institut Elie Cartan, UMR 7502 UHP CNRS INRIA, Université Henri Poincaré (NANCY 1), 54506 VANdeunre-LÈs-NANCY, France

E-mail: wujie@iecn.u-nancy.fr 